\title{
Lambda-type Three-Level Laser Coupled to Squeezed Vacuum Reservoir
}

\author{
Takele Teshome, Gomera Biyazn, Misrak Gatahun
}

Department of Physics, Hawassa University, Hawassa, Ethiopia

Email address:

biyazngomera17@gmail.com (G. Biyazn)

\section{To cite this article:}

Takele Teshome, Gomera Biyazn, Misrak Gatahun. Lambda-type Three-Level Laser Coupled to Squeezed Vacuum Reservoir. Optics. Vol. 10, No. 1, 2021, pp. 1-6. doi: 10.11648/j.optics.20211001.11

Received: March 7, 2021; Accepted: March 17, 2021; Published: March 30, 2021

\begin{abstract}
The squeezing, entanglement, and statistical properties of light produced by lambda-type three-level laser coupled to squeezed vacuum reservoir have been studied. We have determined the quadrature variances mean as well as variance photon number photon for cavity modes with the aid of the solutions of c-number Langevin equations associated with the normal order. The results show that the light produced by the system under consideration in squeezed state. The squeezed vacuum reservoir enhances both the quadrature squeezing and mean photon numbers. In addition, the mean photon number of mode $b$ is greater than that of a if more atoms injected with state $\mid a>$. We have calculated the quadrature variances of the cavity modes, the result shows that the light produced by the system under consideration is in squeezed state and the squeezing occurs in the minus quadrature. The result also shows that the effect of the squeezed vacuum reservoir is not only enhancing the degree of squeezing for the light modes but also to generate the photons for the system under consideration. The system produce entangled two mode light that increase with $\eta$ and linear gain coefficient but decrease with squeezing parameters. The correlation function of the two modes decreases as $\eta$, the linear gain coefficient and increase with squeezing parameter.
\end{abstract}

Keyword: Entanglement, EPR, LASER, Photon Statistics

\section{Introduction}

Quantum optics is a field of quantum physics that deals with the interaction of radiation with matter. [2] The squeezing and Statistical properties of the radiation generated by different quantum optical systems have been investigated since the earliest days that quantum optics was known. The quantum description of radiation is one of the central topics in quantum optics.

This description requires the quantization of the radiation field. The quantization of the radiation field leads to the introduction of various possible quantum states of light such as the number, the coherent, chaotic, and the squeezed states.

Squeezing is one of the non-classical features of light that has attracted a great deal of interest. In squeezed light, the noise in one quadrature is below the coherent or vacuum state level at the expense of enhanced fluctuations in the other quadrature with the product of the uncertainty in the two quadrature's satisfying the uncertainty relation. [1, 3-13] Squeezed light has potential application in low noise optical communication and weak signal detection.
A three-level laser may be defined as a quantum optical system in which three-level atoms, initially prepared in a coherent superposition of the two levels, are injected into a cavity. When a three-level atom makes a transition from top to the bottom levels, two photons are generated. The coupling of the two levels of the atom is responsible for the interesting non classical features of the generated light. In general, the atomic coherence can be induced in a three-level atom by coupling with coherent light or by preparing the atom initially incoherent superposition of these two levels. There are several quantum optical systems that could generate light with non-classical features such as squeezing, entanglement, and anti-bunching etc.

Entangled states can be used in quantum cryptography, quantum teleportation, super dense coding, and, quantum computation etc. Quantum entanglement also represents in the dispensable physical resource for the description and performance of quantum information processing task. Thus a great deal of efforts have been developed to study and characterizing entanglement in the recent years. The central task of quantum information theory is to characterize and 
quantify entanglement of a given system.

\section{Cavity Mode Variables}

We first drive the Hamiltonian describing the interaction of a radiation with lambda-type three-level atom seen in Figure 1 and then the master equation for three-level laser coupled to squeezed vacuum reservoir.

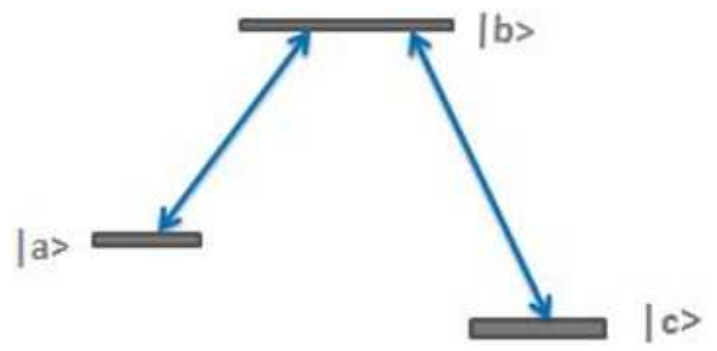

Figure 1. Schematic representation of a three-level atom in a lambda configuration.

\subsection{The Quantum Hamiltonian}

The interaction of a one-electron atom having charge e and mass $m$ with a two-mode radiation represented by the vector potential $\mathrm{A}$ is described by the Hamiltonian

$$
\begin{gathered}
\hat{\mathrm{H}}_{I}=-e \hat{\mathrm{r}} \frac{d}{d t} \hat{\mathrm{A}} \\
\hat{\mathrm{A}}=A\left(\hat{\mathrm{a}} e^{-i \omega_{a} t}+\hat{\mathrm{a}}^{+} e^{i \omega_{a} t}\right)+B\left(\hat{b} e^{-i \omega_{b} t}++\hat{b}^{+} e^{i \omega_{b} t}\right) \hat{u}
\end{gathered}
$$

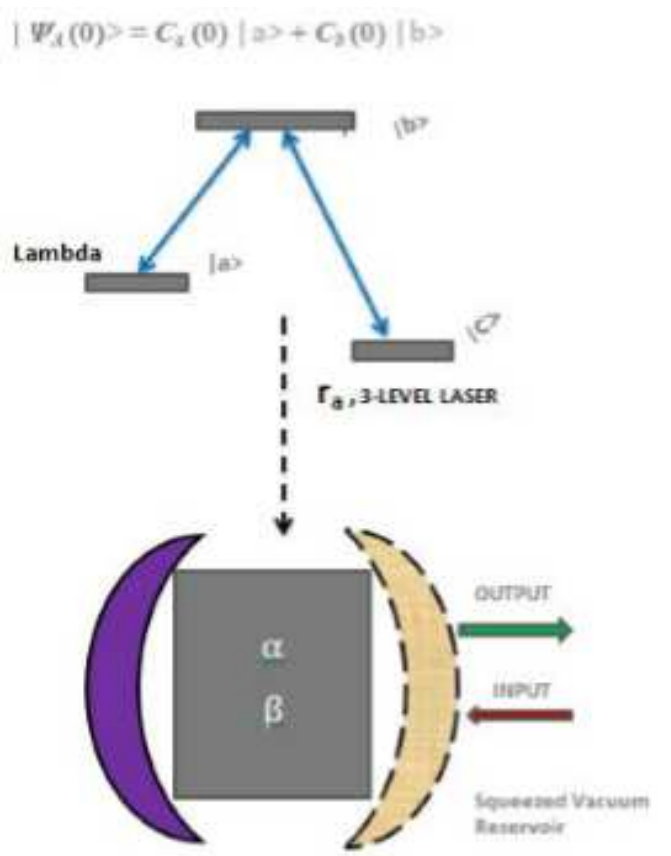

Figure 2. Schematic representation of three-level laser with squeezed vacuum reservoir

\subsection{The Master Equation}

We consider a three-level lasers in which three-level atoms in $\Lambda$ configuration are injected into the cavity at a constant rate $r_{a}$ and removed from the cavity after a certain time $\tau$. We denote levels of three-level atom by $|a>| b>$,, and $|c\rangle$. We assume that the cavity modes to be at resonance with the two transitions $|b>\leftrightarrow| a>$ and $|b>\leftrightarrow| c>$ are dipole allowed and direct transition between levels $|a>\leftrightarrow| c>$ to be dipole forbidden as shown in Figure 2 The interaction of $\Lambda$-type three-level atom with the cavity modes can be described in the interaction picture by the Hamiltonian

$$
H_{I}=\operatorname{ig}\left(\hat{\sigma}_{b a} \hat{a}-\hat{\sigma}_{a b} \hat{a}^{+}+\hat{\sigma}_{b c} \hat{b}-\widehat{\sigma}_{c b} \hat{b}^{+}\right)
$$

We take the initial state of a three-level atom to be

$$
\psi_{\mathrm{A}}(0)=\mathrm{c}_{\mathrm{a}}(0)\left|\mathrm{a}>+\mathrm{c}_{\mathrm{c}}(0)\right| \mathrm{c}>
$$

The master equation for the cavity modes produced by a $\lambda$ type three-level laser whose cavity modes are coupled by a two-mode squeezed vacuum can be written in the form

$$
\begin{aligned}
& \frac{d}{d t} \rho=\frac{1}{2}\left(k(N+1)+A \rho_{a a}^{0}\right)\left(2 \text { â } \rho \mathrm{a}^{+}-\mathrm{a}^{+} \mathrm{a} \rho-\rho \mathrm{a}^{+} \mathrm{a}\right)+ \\
& \left.\frac{1}{2} k N\left(2 \mathrm{a}^{+} \rho \mathrm{a}-\mathrm{a} \mathrm{a}^{+} \rho-\rho \mathrm{a} \mathrm{a}^{+}\right)\right)+\frac{1}{2}\left(k N+A \rho_{C C}^{0}\right) \\
& \left(2 \hat{b} \rho \hat{\mathrm{b}}^{+}-\hat{\mathrm{b}}^{+} \hat{b} \rho-\rho \hat{\mathrm{b}}^{+} \hat{b}\right)+\frac{1}{2} k N\left(2 \hat{\mathrm{b}}^{+} \rho \hat{b}-\hat{b} \hat{\mathrm{b}}^{+} \rho-\right. \\
& \left.\rho \hat{b} \hat{\mathrm{b}}^{+}\right)+\frac{1}{2}\left(A \rho_{a c}^{0}\right)\left(2 \mathrm{a} \rho \hat{b}^{+}-\rho \hat{b}^{+} \hat{\mathrm{a}}-\hat{b}^{+} \mathrm{a} \rho+2 \hat{b} \rho \hat{a}^{+}-\right. \\
& \left.\rho \mathrm{a}^{+} \hat{b}-\mathrm{a}^{+} \hat{b} \rho\right)++1 / 2 k M\left(2 \hat{a}^{+} \hat{b}^{+} \rho-\hat{a}^{+} \hat{b}^{+} \rho-\rho \hat{a}^{+} \hat{b}^{+}+2 \hat{\mathrm{a}} \widehat{b} \rho-\right. \\
& \text { â } \widehat{b} \rho-\rho \text { â } \widehat{b})+\frac{1}{2} k M\left(2 \hat{b}^{+} \hat{a}^{+} \rho-\hat{a}^{+} \hat{b}^{+} \rho-\rho \hat{a}^{+} \hat{b}^{+}+2 \widehat{b} \mathrm{a} \rho-\right. \\
& \text { â } \widehat{b} \rho-\rho \hat{a} \widehat{b})
\end{aligned}
$$

\subsection{C-number Langevin Equations}

The c- number langevin equation associated to the normal orders.

$$
\begin{gathered}
\frac{d}{d t}<\alpha>=-\frac{1}{2} \mu_{a}<\alpha>-\frac{1}{2} v<\beta> \\
\frac{d}{d t}<\beta>=-\frac{1}{2} \mu_{c}<\beta>-\frac{1}{2} v<\alpha> \\
\frac{d}{d t}<\alpha^{2}>=-\mu_{a}<\alpha^{2}>-v<\alpha \beta> \\
\frac{d}{d t}<\beta^{2}>=-\mu_{c}<\beta^{2}>-v<\alpha \beta> \\
\frac{d}{d t}<\alpha^{*} \alpha>=-\mu_{a}<\alpha^{*} \alpha>-\frac{1}{2} v\left(<\alpha^{*} \beta>+<\beta^{*} \alpha>\right)+k N \\
\frac{d}{d t}<\beta^{*} \beta>=-\mu_{c}<\beta^{*} \beta>-\frac{1}{2} v\left(<\alpha^{*} \beta>+<\beta^{*} \alpha>\right)+k N, \\
\frac{d}{d t}<\alpha \beta>=-\frac{1}{2}\left(\mu_{a}+\mu_{c}\right)<\alpha \beta>-\frac{1}{2}<v> \\
\left(<\alpha^{2}>+<\beta^{2}>\right)-k M, \\
\frac{d}{d t}<\alpha^{*} \beta>=-\frac{1}{2}\left(\mu_{a}+\mu_{c}\right)<\alpha^{*} \beta>-\frac{1}{2}<v> \\
\left(<\alpha^{*} \alpha>+<\beta^{*} \beta>\right),
\end{gathered}
$$

Where $\mu_{a}=k+A \rho_{a a}^{0}, \mu_{c}=k+A \rho_{c c}^{0}$ and $v=A \rho_{a c}^{0}=A \rho_{c a}^{0}$. On the base of this equation

$$
\begin{gathered}
\frac{d}{d t}\left\langle\alpha(t)>=-\frac{1}{2} \mu_{a}<\alpha(t)>-\frac{1}{2} v<\beta(t)>+f_{\alpha}(t),\right. \\
\frac{d}{d t}<\beta(t)>=-\frac{1}{2} \mu_{c}<\beta(t)>-\frac{1}{2} v<\alpha(t)>+f_{\beta}(t),
\end{gathered}
$$

Where $f_{\alpha}(t)$ and $f_{\beta}(t)$ are noise forces. The solution of equation (14) and (15) can be written as 


$$
\begin{aligned}
& \alpha(t)=\alpha(0) e^{-\frac{1}{2} \mu_{a} t}+\int d t e^{-\frac{1}{2} \mu_{a}\left(t-t^{\prime}\right)}\left[-\frac{1}{2} v \beta\left(t^{\prime}\right)+f_{\alpha}\left(t^{\prime}\right)\right], \\
& \beta(t)=\beta(0) e^{-\frac{1}{2} \mu_{c} t}+\int d t e^{-\frac{1}{2} \mu_{c}\left(t-t^{\prime}\right)}\left[-\frac{1}{2} v \alpha\left(t^{\prime}\right)+f_{\beta}\left(t^{\prime}\right)\right],
\end{aligned}
$$

The solution of coupled differential equation Eq. (14) and Eq. (15) can be written in the matric form as

$$
\frac{d}{d t} Y(t)=-\frac{1}{2} M Y(t)+F(t)
$$

Where

$$
\begin{array}{r}
Y(t)=\begin{array}{l}
\alpha(t) \\
\beta(t)
\end{array} \\
\mathrm{M}=\begin{array}{cc}
\mu_{a} & v \\
v & \mu_{c}
\end{array} \\
F(t)=\begin{array}{l}
f_{\alpha}(t) \\
f_{\beta}(t)
\end{array}
\end{array}
$$

We next proceed to find the eigenvalues and eigenvectors of the matrix M. Applying the eigenvalue equation

$$
M V i=\lambda V i,
$$

we find the characteristic equation

$$
\lambda^{2}-(\mu a+\mu c) \lambda+\left(\mu_{a} \mu_{c}-v^{2}\right)=0
$$

the relation,

$$
\rho_{a a}^{(0)}+\rho_{c c}^{(0)}=1
$$

Then the Eigen value

$$
\lambda \pm=\frac{1}{2}(2 \kappa+A \pm A)
$$

\section{Quadrature Fluctuation}

\subsection{Quadrature Variances}

The squeezing properties of the two-mode light in the cavity can be described by two the quadrature squeezing property of two-mode light is described by two Hermitian quadrature operators defined by

$$
\begin{array}{r}
\hat{c}_{ \pm}=\sqrt{ \pm 1}\left(\widehat{c}^{+} \pm \hat{c}\right), \\
\Delta c_{ \pm}^{2}=1 \pm<\gamma_{ \pm}, \gamma_{ \pm}>,
\end{array}
$$

Where

$$
\begin{aligned}
& \gamma_{ \pm}=\gamma^{*} \pm \gamma \\
& \gamma=\frac{1}{\sqrt{2}}(\alpha+\beta) .
\end{aligned}
$$

With the aid of (29) and (28) we have written Eq. (27) as

$$
\begin{gathered}
\Delta \hat{c}^{2}{ }_{ \pm}=1+<\alpha^{*} \alpha>+<\beta^{*} \beta>+2<\alpha^{*} \beta> \pm<\alpha^{2}> \\
\pm<\beta^{2}> \pm 4<\alpha \beta> \\
\Delta \hat{c}^{2}{ }_{ \pm}=\frac{(2 \kappa+A)^{2}(1+N \pm 2 M)-2 v(2 \kappa+A)(N \pm M)-\kappa(2 \kappa+A) \mp 2 M A^{2} \eta^{2}}{(2 \kappa+A)(\kappa+A)}
\end{gathered}
$$

Eq. (31) represents the quadrature variance of the twomode light produced by lambda type three-level laser coupled to squeezed vacuum reservoir.

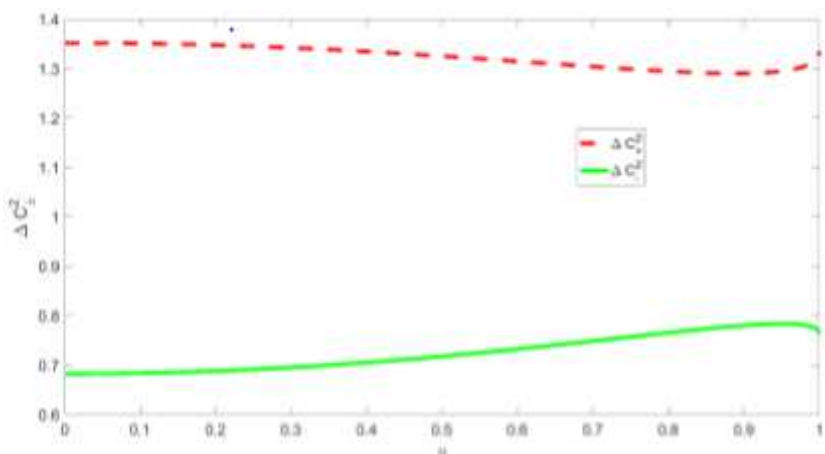

Figure 3. Plots of the quadrature variances Eq. (31)) versus $\eta$ for $A=3, \kappa=$ 0.8 , and $r=0.2$.

Figure 3 represents the plots of the plus and minus quadrature variances Eq. (31) versus $\eta$ for $A=3, \kappa=0.8$, and $\mathrm{r}=0.2$. The figure shows that minus quadrature variance is less than quadrature variance of coherent light.

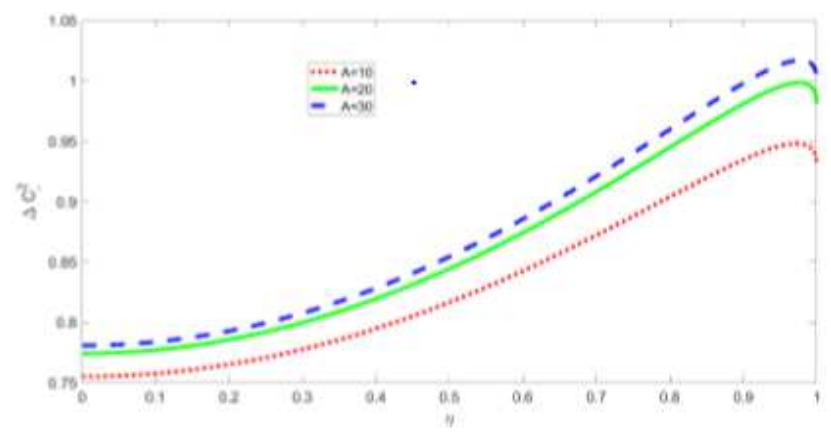

Figure 4. Plots of the minus quadrature variance versus $\eta$ for $\kappa=0.8, r=$ 0.2 and for different values of the linear gain coefficient and squeezing parameter.

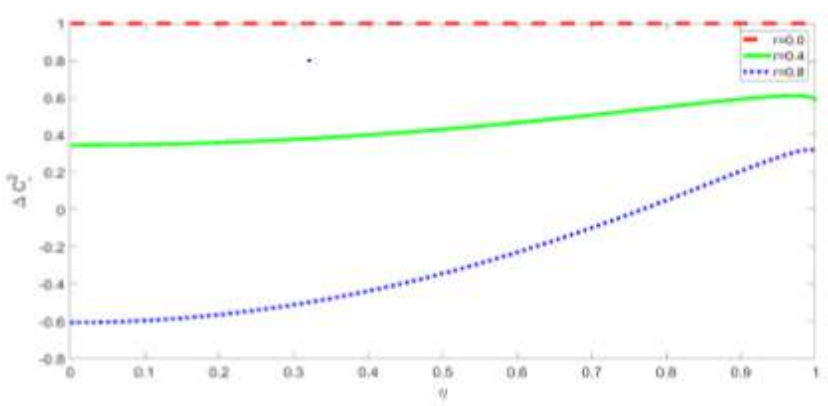

Figure 5. Plots of the minus quadrature variance versus $\eta$ for $\kappa=0.8, A=$ 10 and for different value of squeezing parameter.

We see from Figures 4 and 5 the minus quadrature variance increase with $\eta$ and linear gain coefficient but decrease with squeezing parameter. The quadrature squeezing decrease with linear gain coefficient and increase with squeezing parameter.

We now consider the case in which the cavity mode is coupled to a two-mode vacuum reservoir. Then up on setting $r=0$ in Eq. (31), we get 


$$
\Delta c_{-}^{2}=1
$$

This is the quadrature variances of the cavity modes for lambda-type three-level initially prepared in the coherent superposition of the lower levels.

\subsection{Entanglement}

In this section we seek to determine the entanglement properties of the two-mode light produced by $\lambda$-type threelevel laser.

$$
\Delta u^{2}+\Delta v^{2}=2+2<\alpha^{*} \alpha>+2<\beta^{*} \beta>-4<\alpha \beta>
$$

the sum of the variances of the two EPR-like variables takes the form

$$
\Delta u^{2}+\Delta v^{2}=2+\frac{(N-M)(2 \kappa+A)^{2}+M A^{2} \eta^{2}}{(2 \kappa+A)(\kappa+A)}
$$

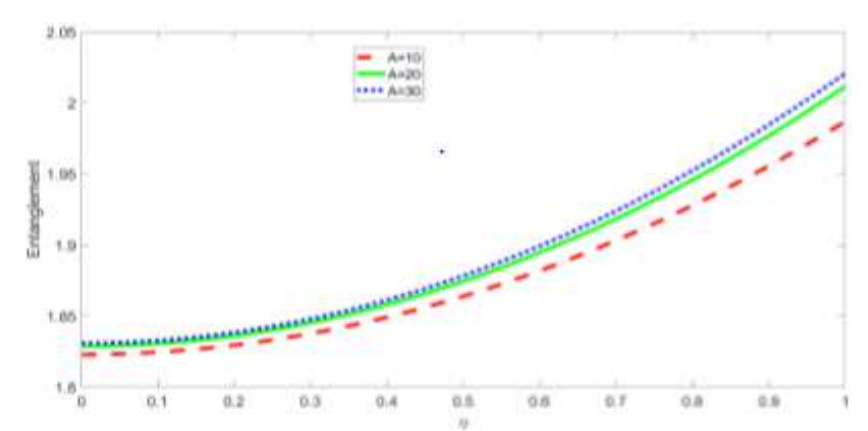

Figure 6. Plots of the sum of the entanglement Eq. (34) versus $\eta$ for $\kappa=0.8, r=0.2$ and for different values of the linear gain coefficient.

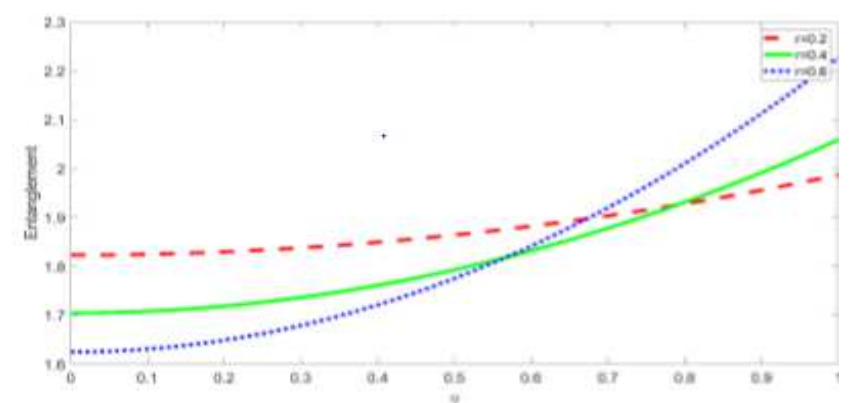

Figure 7. Plots of the entanglement Eq. (34) versus $\eta$ for $A=10, \kappa=0.8$ and for different values of the squeezing parameter.

Eq. (34) represents the sum of the variances of a pair of EPR-like operators for a two mode light produced by $\lambda$-type three-level Lasers with squeezed vacuum reservoir.

Figures 6 and 7 shows that entanglement increase with $\eta$ and linear gain coefficient but decrease with squeezing parameter. The system produces entangled light.

\section{Photon Statistics}

In this chapter, we seek to study statistical properties for the system under-consideration.

Using the solutions of the langevin equation and the correlation properties of the noise forces, we calculate mean and variance of the photon number as well as the photon number correlation of the cavity modes.

\subsection{Mean and Variance of the Photon Number}

The definition of the mean photon number for two-mode light,

$$
\bar{n}=\left\langle\hat{c}^{+} \hat{c}\right\rangle
$$

the mean photon number can be expressed in terms c-number variables as

$$
\bar{n}=\bar{n}_{a}+\bar{n}_{b}+2<\alpha^{*} \beta>,
$$

where $\bar{n}_{a}=\left\langle\alpha^{*} \alpha\right\rangle$ and $\bar{n}_{\beta}=\left\langle\beta^{*} \beta\right\rangle$ are the mean photon number for mode a and $b$, respectively

$$
\bar{n}=\frac{N(2 \kappa+A-2 v)}{\kappa+A}
$$

We see from Eq. (37), the squeezed vacuum reservoir is not only enhances the mean photon number of the cavity modes it is also the cause of production of photons in the cavity.

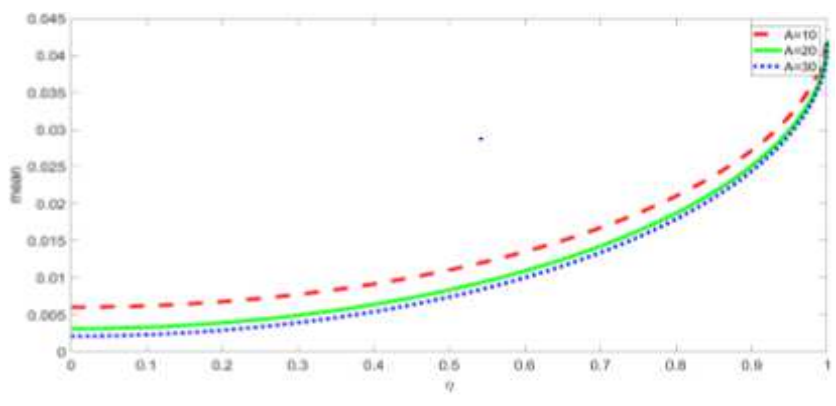

Figure 8. Plot of the mean Eq.(37) versus $\eta$ for $\kappa=0.8$, and $r=0.2$ and different value of $A$.

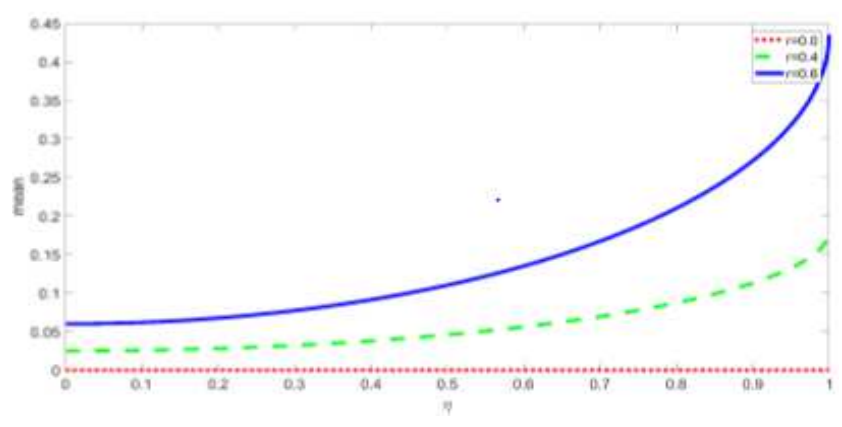

Figure 9. Plot of the mean Eq.(37) versus $\eta$ for $A=10, \kappa=0.8$, and different value squeezing parameter.

Figures 8 and 9 the mean photo number increase with squeezing parameter and $\eta$ but decrease with linear gain coefficient.

We next proceed to determine the mean photon number sum and difference. We define the mean photon number sum and difference as

$$
\begin{gathered}
\bar{n} \pm=\bar{n}_{a} \pm \bar{n}_{b} . \\
n \pm=\frac{N((1 \pm 1)(2 \kappa+A)-(1 \mp 1) A \eta)}{2(\kappa+A)}
\end{gathered}
$$

This equation represents the mean photon number sum and difference for lambda-type three level laser coupled to squeezed vacuum reservoir. We see from Eq. (39) that the 
mean of the photon number difference is negative. This shows that the mean photon numbers of mode $b$ is greater than that of mode $a$. This must be photons with mode a have more probability to be absorbed by the atoms.

We now consider the case in which the reservoir is ordinary vacuum. Thus up on setting $r=0$ in Eq. (39), we get

$$
\bar{n}_{ \pm}=0
$$

We next proceed to calculate the variance of the photon number. The variance of the photon for two-mode light defined by

$$
\Delta n^{2}=<\hat{n}^{2}>-<\hat{n}>^{2}
$$

can be put in the form

$$
\Delta n^{2}=\Delta n_{a}^{2}+\Delta n_{b}^{2}+2<\alpha^{*} \alpha \beta^{*} \beta>-2 \bar{n}_{a} \bar{n}_{b}
$$

in which

$$
\Delta n_{a}^{2}=<\alpha^{* 2} \alpha^{2}>+\bar{n}_{a}-\bar{n}_{a}^{2}
$$

is the photon number variance for mode $a$ and

$$
\Delta n_{b}^{2}=<\beta^{* 2} \beta^{2}>+\bar{n}_{b}-\bar{n}_{b}^{2}
$$

the variance of two-mode light can be written

$$
\Delta n^{2}=\bar{n}^{2}+\bar{n}_{a}+\bar{n}_{b}+|<\alpha+\beta>|^{2},
$$

We see from Eq. (45) greater than the mean photon number. This indicates that it exhibits super Poissonian statistics.

\subsection{Photon Number Correlation}

The second order photon number correlation can be defined as

$$
\begin{gathered}
g_{a b}^{(2)}(0)=\frac{<\hat{n}_{a} \hat{n}_{b}>}{<\hat{n}_{a}><\hat{n}_{b}>} \\
g_{a b}^{(2)}(0)=1+\frac{2 v M}{(2 \kappa+A) N}
\end{gathered}
$$

The result in Eq. (47) shows that the photon number correlation is greater than one. This indicates that there is some correlation between mode $a$ and mode $b$. This inter mode correlation must be due to the atomic coherence induced by atoms initially prepared in a coherent superposition of the bottom levels.

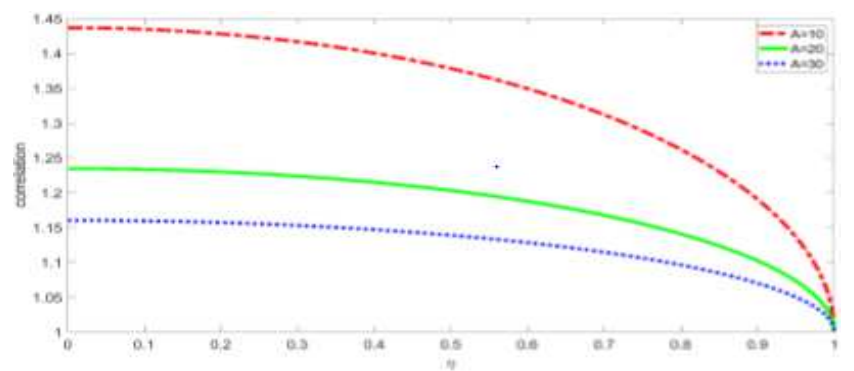

Figure 10. Plots of the correlation Eq.(47) for $\kappa=0.8, r=0.2$ and for different value of linear gain coefficient.

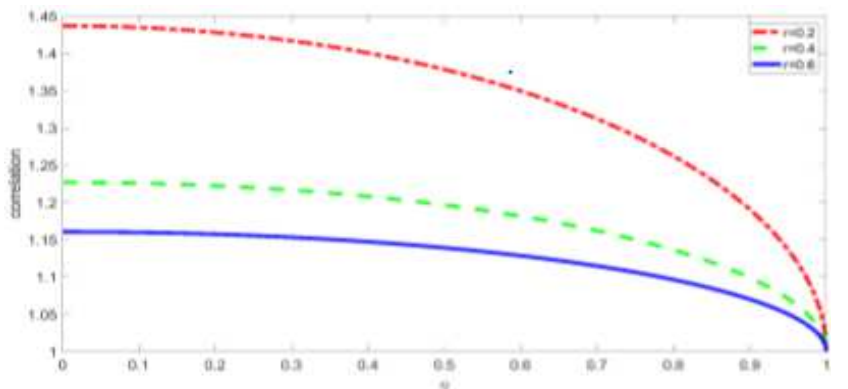

Figure 11. Plots of the correlation Eq. (47) for $\kappa=0.8, A=10$ and for different value of squeezing parameter.

\section{Conclusion}

In this thesis the squeezing, entanglement and statistical properties of the light generated by a lambda-type threelevel laser coupled to squeezed vacuum reservoir have been analyzed applying the solutions of the c- number langavin equation associated with the normal order. We have calculated the quadrature variances of the cavity modes. Our result shows that the light produced by the system under consideration is in squeezed state and the squeezing occurs in the minus quadrature. The result also shows that the effect of the squeezed vacuum reservoir is not only enhancing the degree of squeezing for the light modes but also to generate the photons for the system under consideration. The system produce entangled two mode light that increase with eta and linear gain coefficient but decrease with squeezing parameters. The correlation function of the two modes decreases as $\eta$, the linear gain coefficient and increase with squeezing parameter. The mean of the photon number increase with squeezing parameter but decrease with linear gain coefficient. In general, the light produce by the system exhibits super poissonian photon statistics.

\section{References}

[1] Fesseha Kassahun, Fundamental of Quantum Optics (Lulu, Press Inc., North Carolina, 2008).

[2] Marlin O. Scull y and M. Suhail Zubairy, Quantum Optics (Cambridge University, 1997).

[3] Mark Fox, Quantum Optics An Introduction, (Oxford University press, 2006).

[4] Fesseha K. Three-level laser can generate squeezed light (Addis Ababa University, Ethiopia, 2005).

[5] E. Alebachw and K. Fesseha, A degenerate three-level laser with a parametric amplifier, (Addis Ababa University. V1, 2008).

[6] Dawit Hiluf, A Degenerated three-level laser coupled to a squeezed vacuum reservoir, (Addis Ababa University, 2005).

[7] F. Kasshun, Three level laser dynamics with the atoms pumped by electron bombardment (Addis Ababa, Ethiopia. v3, 2012). 
[8] Misrak Getahun, Three level laser Dynamics with coherently and squeezed light, $\mathrm{PhD}$ Dissertation (Addis Ababa University, 2009).

[9] S. Tesfa driven degenerate three level cascade laser, (Phys. B: At. Mol. Opt. Phys. 41, 145501 (2008)).

[10] K. fesseha, Three -level laser dynamic with squeezed light, (10.1103 PhysRevA.63.033811, published 13 February 2001).
[11] Javaid Anwar, Laser phase noise quenching via squeezed vacuum reservoir, (PhysRevA72, 063804 2005).

[12] Driba Demissie, Quantum analysis of coherently Driven Three level laser Msc. Thesis (Addis Ababa university, 2001).

[13] Wubshet Mekonen, a non degenerate three-level laser with cavity modes driven by coherence light, MSc Thesis, (Addis Ababa University, 2007). 